\title{
CASE OF THE MONTH
}

\section{Lumpy breasts and headache - a crucial ultrasound}

\author{
${ }^{1} S$ HINZE, MRCP, ${ }^{1} Y$ M HART, MD, FRCP and ${ }^{2}$ R F ADAMS, MRCP, FRCR \\ ${ }^{1}$ Department of Neurology, John Radcliffe Hospital, Oxford Radcliffe Hospitals NHS Trust, Oxford UK and ${ }^{2}$ Oxford Breast \\ Imaging Centre, Churchill Hospital, Oxford Radcliffe Hospitals NHS Trust, Oxford UK
}

\author{
Received 3 July 2010 \\ Revised 6 September 2010 \\ Accepted 20 September \\ 2010 \\ DOI: $10.1259 /$ bjr/23418860 \\ (C) 2011 The British Institute of \\ Radiology
}

A 65-year-old female was recalled to a breast assessment clinic 2 weeks after a routine screening mammogram because she had told the mammographer she had noticed a new lump in her right breast. Her mammogram showed mixed density breast tissue but no suspicious findings. The original lump had subsided since the mammogram, but she had noticed further mildly tender lumpiness developing in both breasts and a string-like thickening in her right axillary tail.
On clinical examination, there was mild tender non-suspicious nodularity in both breasts, with no underlying abnormality on ultrasound. However, ultrasound revealed the palpable band in the right axillary tail to be an artery with a $24 \mathrm{~mm}$ segment of circumferential wall thickening (Figure 1).

On further questioning she reported she had been suffering from a frontal headache for 1 month and was generally feeling tired.

What diagnosis could account for these signs and symptoms? What would you do next?

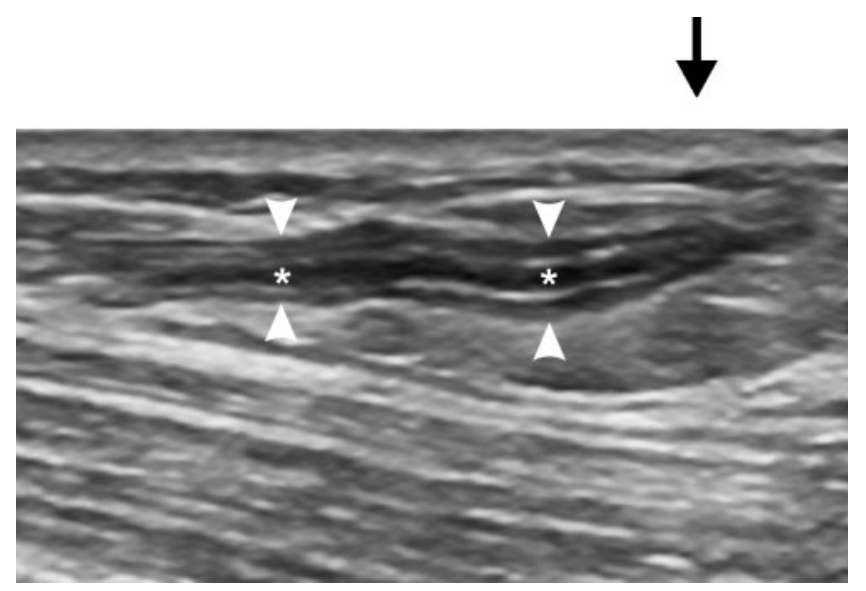

Figure 1. Longitudinal ultrasound image of band-like thickening in right axillary tail, showing "halo sign" (hypoechoic circumferential wall thickening representing oedema) in lateral thoracic artery. Asterisks denote lumen of artery and arrowheads denote exterior of wall. 


\section{Diagnosis}

Giant cell arteritis was suspected by the radiologist. Examination of the patient's temporal arteries revealed them to be nodular and mildly tender. She was referred immediately for assessment by the neurology team. Her erythrocyte sedimentation rate (ESR) and C-reactive protein (CRP) were checked en route to the neurologist and were $89 \mathrm{~mm} \mathrm{~h}^{-1}$ and $23 \mathrm{mg} \mathrm{l}^{-1}$, respectively. She was immediately prescribed $60 \mathrm{mg}$ prednisolone per day. A temporal artery biopsy the following day confirmed the diagnosis of giant cell arteritis (GCA). Her symptoms rapidly improved over the following 2 weeks, including her breast lumps.

\section{Discussion}

This patient met all five American College of Rheumatology (ACR) criteria for GCA i.e. over 50 years, new onset headache, temporal artery tenderness or decreased pulse, ESR $>50 \mathrm{~mm} \mathrm{~h}^{-1}$ and positive temporal artery biopsy. The presence of 3 or more criteria yields a sensitivity of $93.5 \%$ and specificity of $91.2 \%$ [1].

GCA has an insidious onset and is often diagnosed late, after major cardiovascular sequelae have occurred. There are a few reports of GCA causing breast lumps, which are usually tender [2]. In many of these cases the diagnosis was only made after, potentially unnecessary, surgical excision. Breast pain is uncommon in postmenopausal women, but in the era of "breast awareness" tender breast lumps may be the presenting symptom of some women with GCA.

GCA typically affects branches of the carotid and subclavian arteries, and sometimes the aorta and femoropopliteal arteries [1]. The two main arteries supplying the breast, the lateral thoracic artery and the internal mammary artery are both branches of the subclavian artery. The lateral thoracic artery branch in this woman showed the "halo" sign, a hypoechoic circumferential wall thickening representing oedema [3].

Temporal artery biopsy may be negative in patients with a clinical diagnosis of GCA owing to the segmental nature of the inflammation $[1,4,5]$. Ultrasonography of the temporal arteries is used in some centres as an adjunctive diagnostic method and to select a biopsy site
[3]. A recent meta-analysis of temporal artery ultrasonography showed presence of the halo sign had a sensitivity of $69 \%$ and specificity of $82 \%$ when compared with biopsy. When the features of stenosis or occlusion were added in, the combined sensitivity was $88 \%$ [4]. In a few centres with expertise in temporal artery ultrasonography, it has replaced biopsy in patients with a typical presentation of GCA [3,5]. High resolution contrast-enhanced MRI has also been used to demonstrate arterial wall thickening [1,3]. Fluorodeoxyglucose positron emission tomography shows large vessel involvement which can be asymptomatic $[1,3]$.

It is important that the incidental ultrasound finding of a thickened arterial wall should lead to immediate further action. A more detailed history and examination should be sought with regards to signs and symptoms of giant cell arteritis, and inflammatory markers should be checked. Same day referral for specialist clinical assessment and treatment is required to prevent irreversible visual loss and other serious vascular sequelae [1], even if the finding is unexpected.

\section{Acknowledgment}

We would like to thank the patient for readily giving her permission to report her case. She hopes this might facilitate appropriate treatment of other women in a similar situation.

\section{Reference}

1. Salvarani C, Cantini F, Hunder GG. Polymyalgia rheumatica and giant-cell arteritis. Lancet 2008;372:234-45.

2. Marie I, Audeguy P, Francois A, DE Kergal F, Richard C. Giant cell arteritis presenting as a breast lesion: report of a case and review of the literature. Am J Med Sci 2008;335: 489-91.

3. Blockmans D, Bley T, Schmidt W. Imaging for large-vessel vasculitis. Curr Opin Rheumatol 2009;21:19-28.

4. Karassa FB, Matsagas MI, Schmidt WA, Ioannidis JP. Metaanalysis: test performance of ultrasonography for giant-cell arteritis. Ann Intern Med 2005;142:359-69.

5. Alberts MS, Mosen DM. Diagnosing temporal arteritis: duplex vs. biopsy. QJM 2007;100:785-89. 